This article has been accepted as Kohn JC, Abdalrahman T, Sack KL, Reinhart-King CA, Franz T. Cell focal adhesion clustering leads to decreased and homogenized basal strains. Int J Numer Meth Bio, 2019, DOI: 10.1002/cnm.3260

Research Article - Application

\title{
Cell focal adhesion clustering leads to decreased and homogenized basal strains
}

Julie C. Kohn ${ }^{\mathrm{a}, \mathrm{b}}$, Tamer Abdalrahman ${ }^{\mathrm{b}, \mathrm{c}}$, Kevin L. Sack ${ }^{\mathrm{b}}$, Cynthia A. Reinhart-King ${ }^{\mathrm{a}, \mathrm{d}}$, Thomas Franz $^{\mathrm{b}, \mathrm{e}, *}$

${ }^{a}$ Nancy E. and Peter C. Meinig School of Biomedical Engineering, College of Engineering, Cornell University, Ithaca, New York, USA, jck256@ cornell.edu

${ }^{\mathrm{b}}$ Division of Biomedical Engineering, Department of Human Biology, University of Cape

Town, Observatory, South Africa, thomas.franz@uct.ac.za

c Julius Wolff Institute for Biomechanics and Musculoskeletal Regeneration, Charité, Universitätsmedizin Berlin, Berlin, Germany, tamer.abdalrahman@charite.de

${ }^{\mathrm{d}}$ Department of Biomedical Engineering, School of Engineering, Vanderbilt University, Nashville, Tennessee, USA, cynthia.reinhart-king@ vanderbilt.edu

${ }^{\mathrm{e}}$ Bioengineering Science Research Group, Engineering Sciences, Faculty of Engineering and the Environment, University of Southampton, Southampton, UK, thomas.franz@uct.ac.za

Short Title: Cell strain profiles due to focal adhesion clustering

* Corresponding Author:

Thomas Franz, Department of Human Biology, Faculty of Health Sciences University of Cape Town, Private Bag X3, Observatory 7935

South Africa, Email: thomas.franz@uct.ac.za 


\begin{abstract}
The subendothelial matrix of the artery is a complex mechanical environment where endothelial cells respond to and affect changes upon the underlying substrate. Our recent work has demonstrated that endothelial cell strain heterogeneity increases on a more heterogeneous underlying subendothelial matrix, and these cells display increased focal adhesion presence on stiffer substrate areas. However, the impact of these grouped focal adhesions on endothelial cell strains has not been explored. Here, we use finite element modeling to investigate the effects of micro-scale stiffness heterogeneities and focal adhesion location and stiffness on endothelial cell strains. Shear stress applied to the apical cell layer demonstrated a minimal effect on cell strain values while substrate stretch had a greater effect on cell strain in the cell-substrate model. The addition of focal adhesions into the computational model (cell-FA-substrate model) predicted a decrease and homogenization of the cell strains. For simulations including focal adhesions, stiffer and more distributed adhesions caused increased and more heterogeneous endothelial cell strains. Overall, our data indicate that cells may group focal adhesions to minimize and homogenize their basal strains.
\end{abstract}

\title{
Keywords
}

Focal adhesion, mechanobiology, in silico, endothelial cell 


\section{Introduction}

Endothelial cells (ECs) interact with the underlying intima tissue through focal adhesion (FA) contacts, which all cells use to sense their environment and can be altered by substrate stiffness $[1,2]$. We have recently demonstrated that FA contacts increase in number on stiffer regions of a heterogenous substrate [3]. FAs can be found as small dots $(0.2-0.5 \mu \mathrm{m})$ near the cell periphery or as larger dashes $(2-10 \mu \mathrm{m}$ long and $\sim 0.5 \mu \mathrm{m}$ wide) centrally in a cell [4]. The locations of FAs in a cell are also important, as the density of cell contact points affects traction forces [5].

Cell stiffness and actin stress fiber arrangements can be modulated by substrate stiffness [6]. Previously, we found that with advanced age, the intima stiffens heterogeneously; matrix stiffness can range from $2-90 \mathrm{kPa}$ within a 100 by $100 \mu \mathrm{m}$ area, which is typically covered by between 3-5 ECs [7]. Cells exhibit complex traction force distributions when placed on heterogeneous stiffness substrates [8]. However, previous in silico [9] and in vitro [10] studies analyzing internal cell stress profiles with FA presence, still use homogeneous substrates. The impact of a highly heterogeneous stiffness substrates on internal cell forces has not been analyzed in vitro due to the difficulty in experimental setup. In addition, previous in silico models of cell-FA-substrate interactions [11-13] also exclude these highly heterogeneous stiffness substrates that we have observed in vivo [7].

Because the arterial intima stiffens heterogeneously with age [7], it is necessary to understand the impact of this heterogeneous stiffening on ECs. Our previous model of an EC placed on a heterogeneous stiffness substrate demonstrated the importance of quantifying cell basal strains because it showed that EC basal strains increase in heterogeneity on a simulated aged arterial intima [14]. However, this previous model lacked the FA attachments found in vivo and the additional analysis that comes with adding FA contacts. Another of our previous works further motivated our current study. In vitro, ECs on heterogeneous substrates display increased presence of FAs on stiff substrate regions [3]. As the intima has significant stiffness heterogeneities in vivo [7], and ECs have been found to create increased FA attachments on stiff substrate regions [3], we wish to further analyze the impacts of these properties on EC strains. Therefore, these previous works motivate the scientific question: for an EC on a substrate with heterogeneous stiffness, how are EC basal strain profiles impacted by FA locations? Here, we 
use standard in silico model parameters to examine a new scientific question. We created a model with direct EC-substrate contacts, one with EC-FA-substrate contacts where the FAs were distributed throughout the basal cell surface, and one with EC-FA-substrate contacts where the FAs were grouped on high substrate stiffness regions. All simulations were run on homogeneous and heterogeneous stiffness substrates. Therefore, our models examine the effects of different FA locations and substrate stiffness heterogeneities on cell strains in concert.

\section{Materials and Methods}

Development of endothelial cell, substrate, and focal adhesion geometries

The single endothelial cell geometry was adapted from a model developed in our previous work [14]. Briefly, C57Bl/6 male mice aortas were extracted, the artery intima was stained for VEcadherin and nuclei. The stained samples were imaged with confocal microscopy and the images were used to construct a single cell geometry in Simpleware ScanIP software (Synopsis Inc., Mountain View, CA USA). We enforced no-slip conditions at interfaces between the nucleus and cytoplasm components in the cell model. The final single endothelial cell geometry (Fig 1A) was meshed with approximately 17,000 C3D4 linear tetrahedral elements (Fig 1B). The substrates were created as 500 by $500 \mu \mathrm{m}$ rectangular prisms, with a depth of $3 \mu \mathrm{m}$ in Abaqus 6.14 (Simulia, Dassault Systèmes, Waltham, MA, USA) software (Fig 1C). Substrates were meshed using 30,000 C3D10 quadradic tetrahedral elements. Focal adhesions were created as $0.5 \mu \mathrm{m}$ high cylinders, with a radius of $1 \mu \mathrm{m}$ based on previous studies $[4,15,16]$ and were meshed with approximately 16 wedge elements per FA (Fig 1D).

\section{Material properties}

Cell material properties were used as in our previous work [14], and are shown in Table 1. Representative compliant (24.5 kPa) and stiff (30 kPa) homogeneous substrates, and a heterogeneous checkerboard pattern substrate $(24.5 / 30 \mathrm{kPa})$, were used as models of the subendothelial matrix based on our previous experimental work (Fig 1E) [7]. As in this previous model, the substrate material properties were taken from ex vivo measurements from a murine subendothelial matrix [7]. For the heterogeneous substrate, the stiffness pattern changed every 10 $\mu \mathrm{m}$, to best model our previous ex vivo study [7]. To test the effect of FA stiffness, we considered two elastic moduli for the focal adhesions, i.e. $1 \mathrm{kPa}$ or $10 \mathrm{kPa}$, which lie on either 
side of the experimentally determined value of $8.8 \pm 1.3 \mathrm{kPa}$ (mean \pm standard deviation) $[17,18]$. As this work aims to analyze the impacts of different FA properties on EC strains when placed on a heterogeneous substrate, data from both $1 \mathrm{kPa}$ and $10 \mathrm{kPa}$ FAs will be presented here.

Table 1: Linear elastic constitutive model properties for the endothelial cell, substrates, and FAs.

\begin{tabular}{|c|c|c|c|}
\hline Material & $\begin{array}{c}\text { Elastic modulus } \\
\mathbf{( k P a )}\end{array}$ & Poisson's ratio & References \\
\hline Cell cytoplasm & 0.775 & 0.495 & {$[11,19,20]$} \\
\hline Cell nuclei & 5.1 & 0.3 & {$[11,19,20]$} \\
\hline Compliant substrate & 24.5 & 0.495 & {$[7]$} \\
\hline Stiff substrate & 30 & 0.495 & {$[7]$} \\
\hline Compliant FAs & 1 & & \\
\hline Stiff FAs & 10 & 0.3 & {$[17,18]$} \\
\hline
\end{tabular}

\section{Finite element model}

Substrate deformation: The substrates were deformed with $14.2 \%$ strain in one direction, as in our previous study [14] to simulate the circumferential strain in a mouse aorta [21]. The side opposite of the stretching was fixed in all directions, and the other two substrate faces were fixed to allow only for unidirectional movement [14]. These boundary conditions were applied no matter the cell-substrate or cell-FA-substrate contact conditions.

EC shear stress: In addition to the substrate deformation, a tangential shear load was placed on the apical surface of the EC at $12 \mathrm{dyn} / \mathrm{cm}^{2}$. This value was chosen based on our previous experimental study of the dual effects of substrate stiffness and shear stress on ECs [22].

Focal adhesion locations: Twenty focal adhesions were placed between the EC and substrate, which is similar to the number used in previous in silico models of cell-FA-substrate interactions $[11,12]$. FA locations were chosen so as to allow for comparisons between randomly distributed 
adhesions (Distributed FAs) and FAs placed in specific locations based on the local substrate stiffness (Grouped FAs). Previous work from our lab demonstrates that FAs preferentially occur in areas of high stiffness when an EC is placed on a heterogeneous substrate [3]. Therefore, the Grouped FA locations were determined by moving FA locations from the Distributed FA positions to regions within or as close as possible to areas of high substrate stiffness.

Model interactions: For cell-substrate contact models, EC nodes were fixed to the substrate directly, i.e. degrees of freedom directly coupled to match. For models including FAs (cell-FAsubstrate models), the EC nodes were fixed to nodes on the FAs, and nodes on the opposite surface of the FA were fixed to the substrate (Fig 1F). The cell was attached to the substrate (with or without FA contacts) prior to mechanical loading.

A

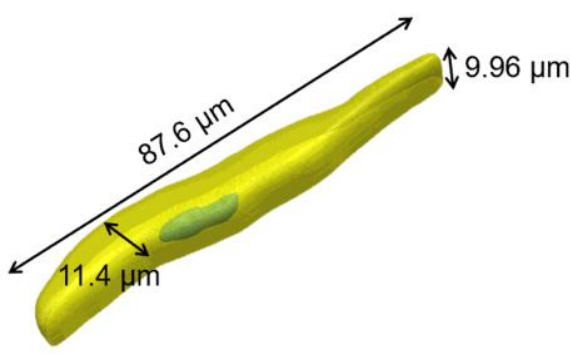

B

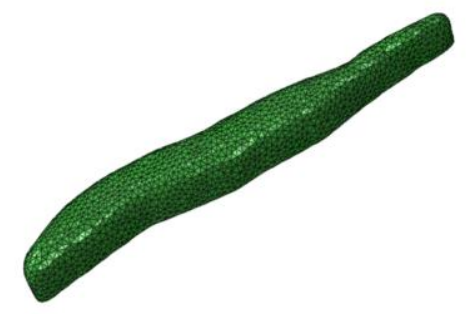

C

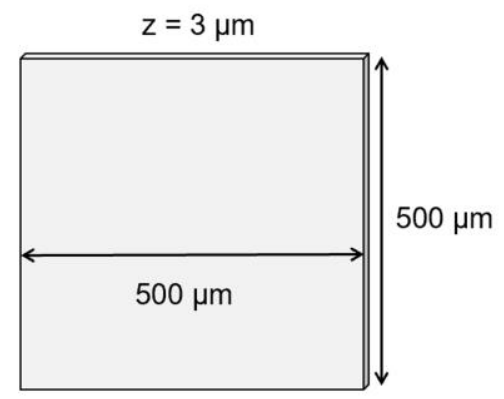

E

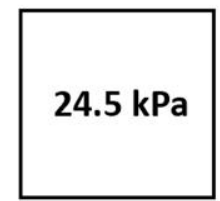

Compliant

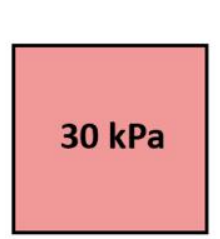

Stiff

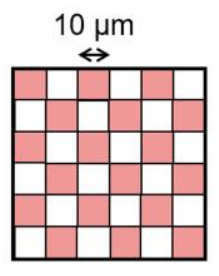

Heterogen.
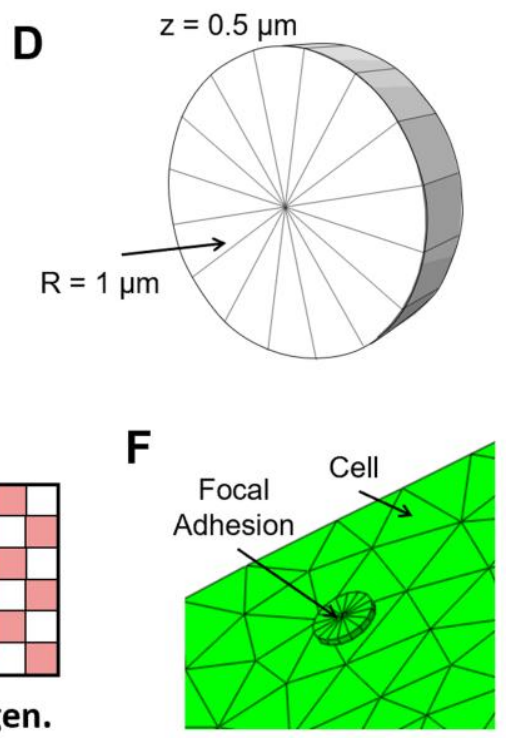

Fig 1: Cell and substrate model parameters. (A) The cell was modeled as $87.6 \mu \mathrm{m}$ long, $11.4 \mu \mathrm{m}$ wide, and with a height of $9.96 \mu \mathrm{m}$, and (B) meshed using C3D4 linear tetrahedral elements. (C) The substrate was modeled as 500 by $500 \mu \mathrm{m}$ with a thickness of $3 \mu \mathrm{m}$. (D) FAs were modeled 
as $0.5 \mu \mathrm{m}$ thick cylinders with a radius of $1 \mu \mathrm{m}$ and meshed with wedge elements. (E) Substrate material properties were used to model a $24.5 \mathrm{kPa}$ compliant substrate, a $30 \mathrm{kPa}$ stiff substrate or a heterogeneous substrate with a checkerboard pattern of both values, where stiffness changes occur every $10 \mu \mathrm{m}$. (F) Example mesh of a FA tied to the EC model.

\section{Data analysis and calculations}

All data were collected following substrate deformation and apical shear stress loading.

Substrate maximum principal logarithmic strain and von Mises stress values were analyzed. For the cell, principal logarithmic strain values were analyzed. Data were exported from each of the element integration points from the models in Abaqus, and then analyzed in Prism 7 (GraphPad, San Diego, CA, USA). To assess heterogeneity profiles, data were analyzed using histogram distributions of strain values. As the histogram data included significant tails, Lorentzian curves were used to fit the data [23], and the Lorentzian curve widths were used as a measure of data heterogeneity.

Percent difference calculations were performed to compare different substrate and FA conditions, as calculated below:

$$
\% \text { Difference }=\frac{\mid \text { value } 1-\text { value } 2 \mid}{\text { average }(\text { value } 1, \text { value } 2)} * 100
$$

\section{Statistical analysis}

To assess normality, the Shapiro-Wilk normality test was used. Non-parametric data were assessed using the Kruskal-Wallis test with Dunn's post-hoc analysis for three comparison groups with non-paired data, and the Friedman test with Dunn's post-hoc analysis for three comparison groups with paired data. Error bars are standard error of the mean (SEM) unless otherwise noted.

\section{Results}

\section{Substrate deformation}

The substrate was deformed with a $14.2 \%$ strain on one end. It was found that compliant and stiff homogenous substrates exhibited uniform strain and stress profiles, while the heterogeneous 
substrate demonstrated a checkerboard pattern of strain and stress (Figs 2 A and B). This model includes the substrate which underwent strain, and does not include the cell. Values were taken from each integration point of the substrate elements. The stiff substrate displayed a higher mean stress than the compliant substrate due to the uniform displacement loading, and the mean stress of the heterogeneous substrate fell between the stress values of the two homogeneous substrates (Fig 2C). Histograms of substrate stresses demonstrated a shift towards higher stress for the stiff substrate and a bimodal stress distribution for the heterogeneous substrate (Fig 2D). These data serve as a proof of concept that the substrates behave as characteristic homogeneous and heterogeneous substrates after displacement and can be used to model different levels of substrate heterogeneity.
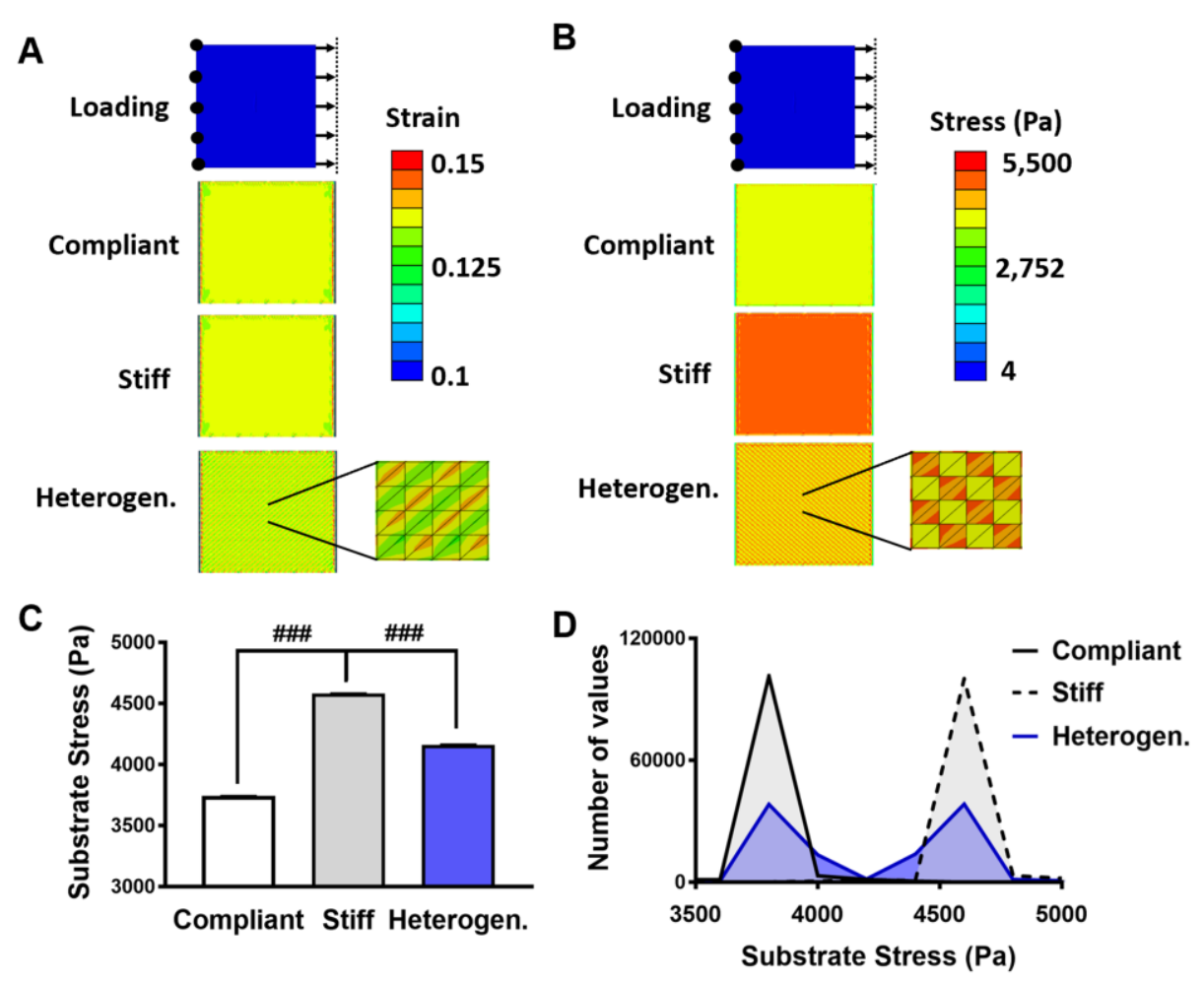

Fig 2: Stretched characteristic substrate models with no cell attached demonstrate change in substrate stress with different stiffness and levels of heterogeneity. (A) The left side of the substrate was fixed (represented by the black circles) and the right side was stretched. The homogeneous compliant and stiff substrates exhibit the same strain profiles due to the uniform uniaxial displacement, while the heterogeneous substrate displays a checkerboard-patterned strain profile. (B) Substrate stresses are uniform on the homogeneous substrates while the 
heterogeneous substrate exhibits its checkerboard pattern. (C) von Mises stress values taken from the integration points of the elements were compared between substrates. Mean substrate stress increases on the stiff substrate and decreases on the compliant substrate, compared to the heterogenous substrate. Error bars are SEM, \#\#\# p < 0.0005, Kruskal-Wallis test with Dunn's post-hoc. (D) Histogram plots demonstrate a shift towards increased substrate stress in the stiff substrate and a bimodal distribution for the heterogeneous substrate.

\section{Substrate stretch has a larger effect on cell strain than apical shear stress}

The cell-substrate model was placed on the homogeneous compliant substrate and different loading conditions were applied: uniaxial substrate stretch of $14.2 \%$ applied perpendicular to the long axis of the cell and apical shear flow of $12 \mathrm{dyn} / \mathrm{cm}^{2}$ applied along the long axis of the cell, or each of these loads alone (Fig 3A). These loading conditions were compared in order to best understand the relative effects of each loading condition on the cell strain outputs. To compare each loading condition's effect on the cell strains, the strains from the EC center z-plane were examined. Strain contours of the center z-plane of the cell (Fig 3B) demonstrate the impact of shear or substrate stretch loading conditions. While the cell basal strains will be important to further analysis, in Figure 3C, we compared the cell strains from the entire thickness of the cell in order to analyze the impacts of the loading conditions on all of the cytoplasm and nucleus elements. The impact of shear stress on total cell strain was significantly less than that of substrate stretch, as shown by the 'shear only' cytoplasm strain value that is an order of magnitude lower than conditions with substrate stretch (Fig 3C). In our model, lower strains are present in the nucleus than in the cytoplasm (Fig 3C). These data highlight the importance of substrate stretch for cell strain analysis. 

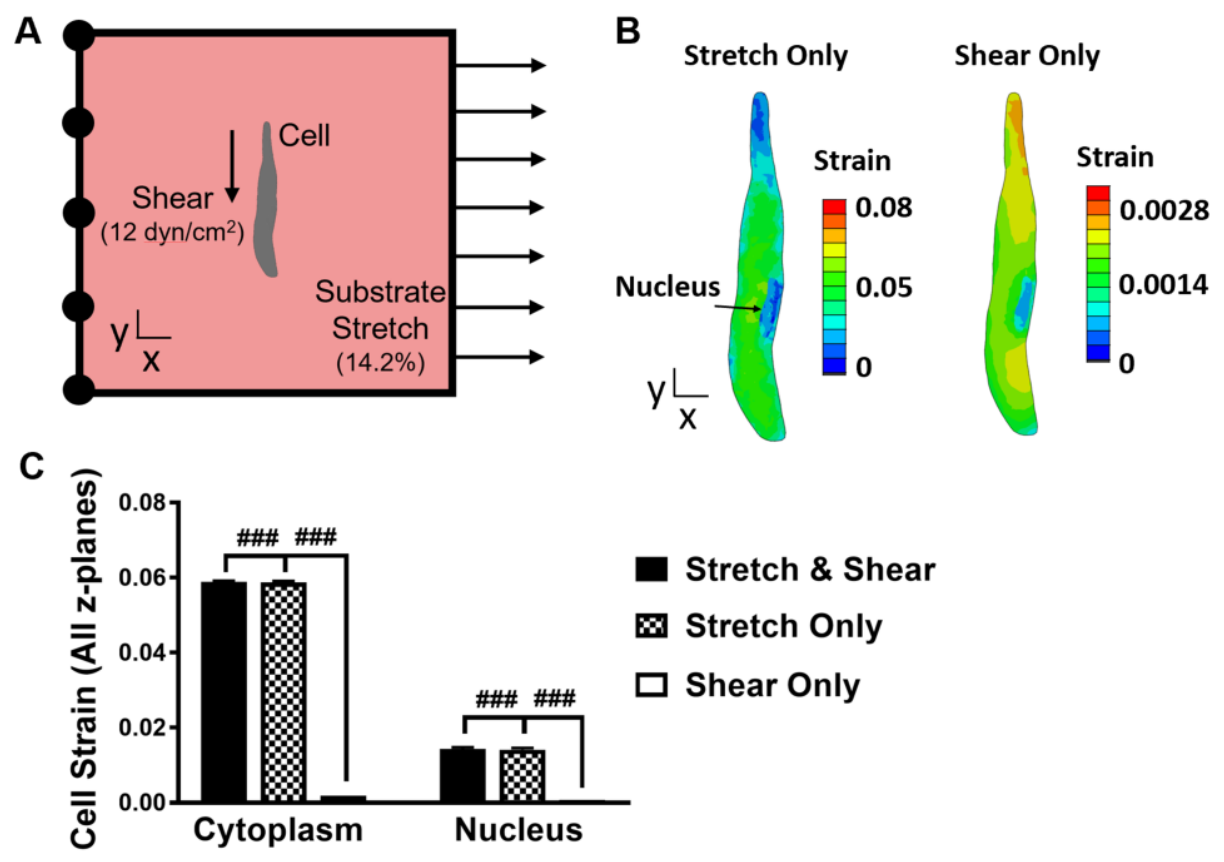

Fig 3: Substrate stretch affects cellular strain more than apical shear stress. (A) Apical shear flow of $12 \mathrm{dyn} / \mathrm{cm}^{2}$ was applied parallel to the direction of cell elongation. Substrate strain of $14.2 \%$ was applied perpendicular to cell elongation while the opposing substrate edge was fixed in place. (B) Cell strain contour plots from the center z-plane demonstrate the lower nuclear strain in both single load conditions. (C) Mean cell strain values from the entire thickness of the cell demonstrating that substrate stretch has a larger effect on cell strain than apical shear stress; values are taken from the cell when placed on the compliant substrate model. Strain values in both the cytoplasm and nucleus demonstrate an order of magnitude difference between shear only and loading conditions with stretch present. Strain values for part C are taken from 17,372 cytoplasm elements and 538 nucleus elements; error bars are SEM, \#\#\# p 0.0005, Friedman test with Dunn's post-hoc.

\section{Cell strain is altered by substrate stiffness heterogeneities (cell-substrate models)}

Cell strains in the basal layer are used to compare the effects of substrate conditions in this study. In the cell-substrate model, the cell basal surface displays higher strain values near the cell edges on all substrate conditions. The cell strain profiles also demonstrate regions of increased or decreased strain in the heterogeneous substrate compared to the homogenous substrates (Fig 4A). The local cell strains are affected by both the cell geometry and also the localized regions of stiff or compliant matrix under the cell in the heterogeneous condition. However, the median basal 
strains do not differ between the substrates (Fig 4B). To compare the heterogeneity of values between each substrate condition, plots were created where the cell basal strain values were separated into ranges and plotted as histograms. As shown in the histogram plots, cell basal strain heterogeneities do occur, and these strains are more pronounced on the heterogeneous substrate (Fig 4C). Lorentzian distributions were fit to the histograms and demonstrate a wider range of cell basal strain values when the cell was placed on the heterogeneous checkerboard substrate compared to the compliant or stiff homogeneous substrates (Fig 4D). The Lorentzian curve width, a measure of heterogeneity, is noticeably elevated for the basal strains of the cell placed on the heterogeneous substrate compared to the homogeneous substrates; the Lorentzian width values showed a $36.8 \%$ and $45.8 \%$ difference from the compliant and stiff homogeneous substrates to the heterogeneous substrate, respectively (Fig 4E). These data demonstrate that heterogeneities present in the substrate translate into cell strain heterogeneities in the cellsubstrate contact models.

A

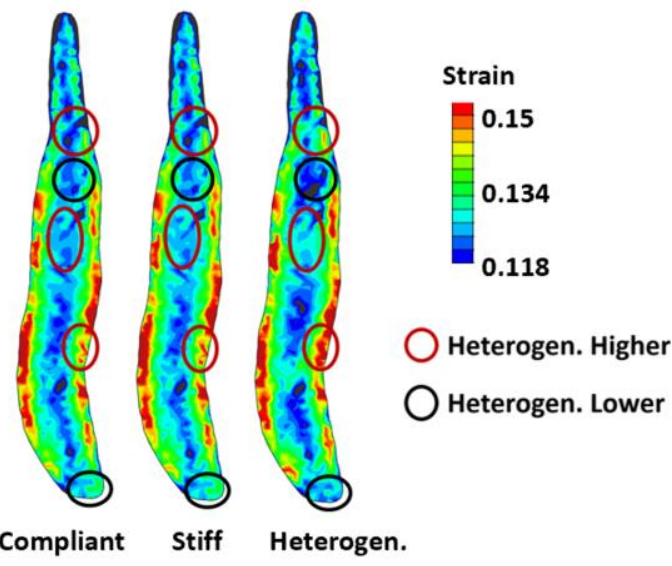

C

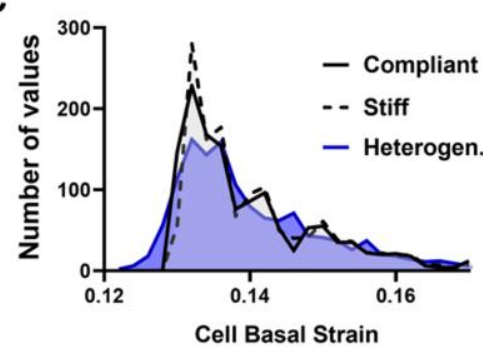

D

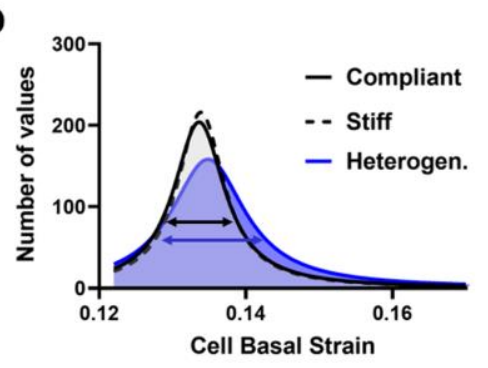

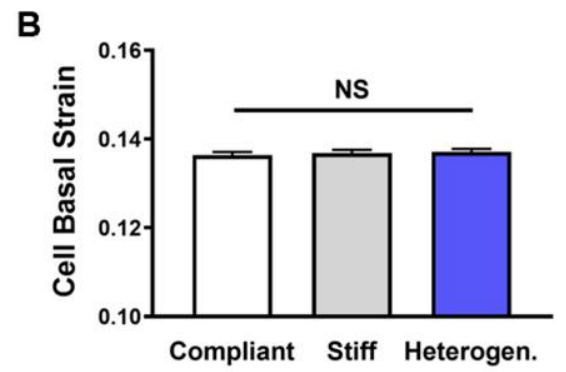

$\mathbf{E}$

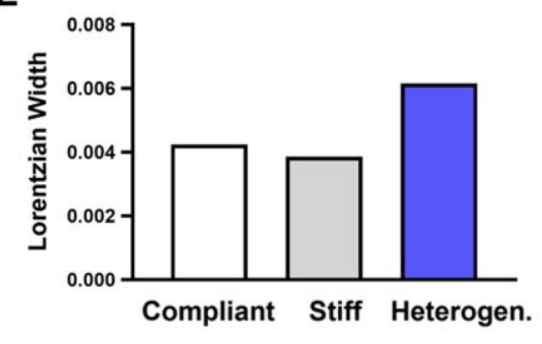

Fig 4: In the cell-substrate contact models following shear and substrate stretch, cell basal strains change due to substrate stiffness heterogeneities. (A) Patterns of basal strain demonstrate higher values near the edges of the single cell. Some areas of the cell display increased (red circles) or 
decreased (black circles) strains on the heterogeneous substrate than on the homogeneous substrates. (B) The median basal strains do not change overall between the substrate conditions; error bars are $95 \%$ confidence interval, NS = not significant (Kruskal-Wallis test). (C) Histogram plots of cell basal strains demonstrate a change in heterogeneity between substrate types, which can be quantified using (D) Lorentzian distributions of cell basal strain; the arrows demonstrate the Lorentzian width measurement. (E) Lorentzian width values demonstrate an increase in heterogeneity in cell basal strains when on the heterogeneous substrate.

\section{Median cell strain is altered by FA stiffness and location (cell-FA-substrate models)}

FAs were placed between the cell and substrate in either a distributed or grouped configuration (Fig 5A). As our previous work indicated,[3] FAs are found in greater abundance on stiff substrate regions; therefore to model the effects of the FA location on cell basal strains, we placed FAs into different configurations. Distributed FAs were dispersed evenly throughout the cell, whereas grouped FAs were primarily located on substrate regions of high stiffness. Cell strains increased with stiff $10 \mathrm{kPa}$ FAs compared to compliant $1 \mathrm{kPa} F A s$, and with the distributed FAs compared to grouped FAs (Figs 5B and 5C). Quantitative analysis confirms these observations (Fig 5D). Median cell basal strain values remain the same between the compliant, stiff, and heterogeneous substrate conditions. Overall, these data indicate that the material properties and locations of the FAs but not the substrate stiffness affect median cellular strains in the cell-FA-substrate contact model. 

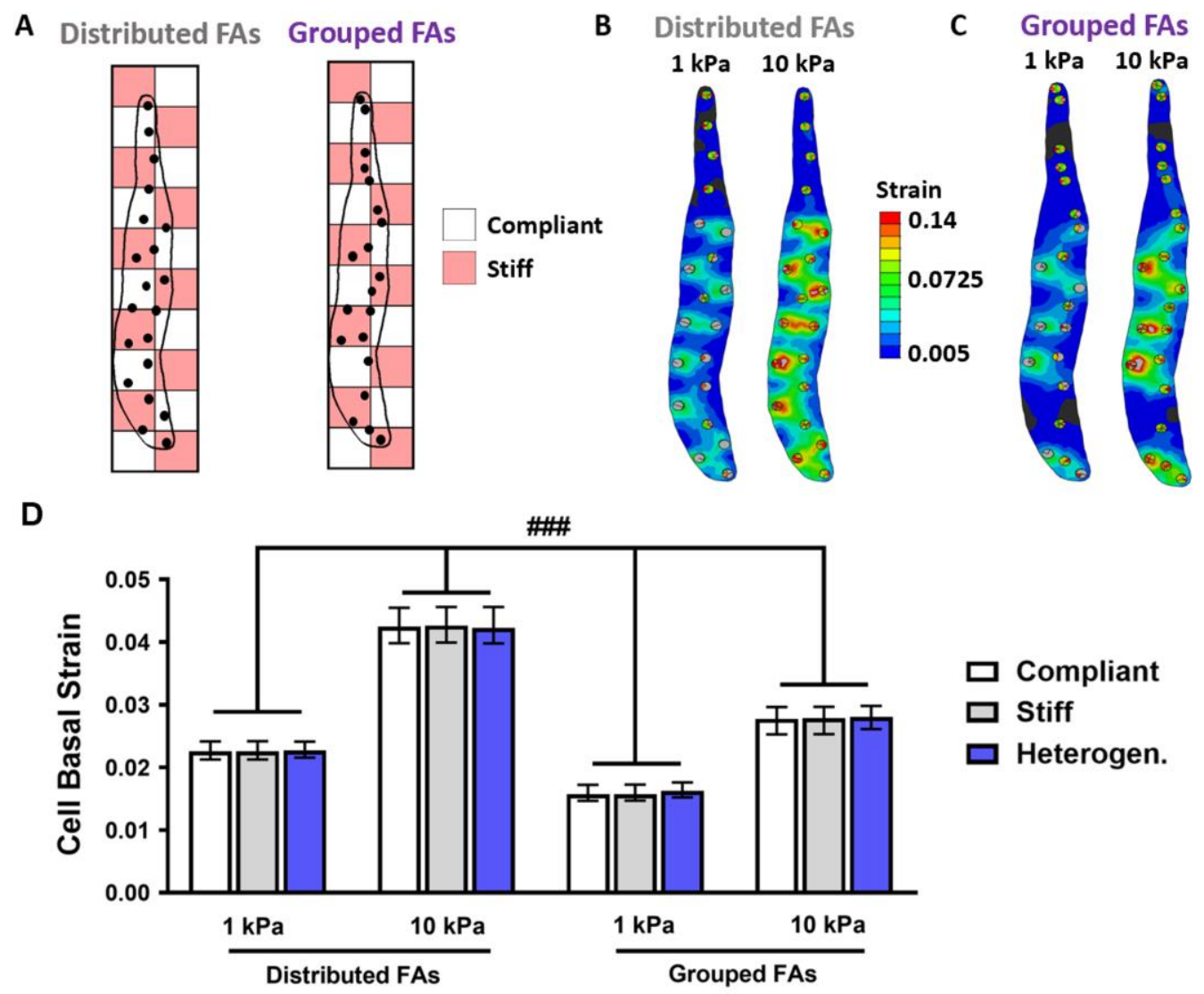

Fig 5: The stiffness and locations of FAs impact cell basal strains. (A) FAs were placed in either 'distributed' or 'grouped' conformations, where the grouped FAs were more likely located on the stiff areas of the heterogeneous checkerboard substrate. (B and C) Strain contour plots of the cell following shear and substrate stretch loading demonstrate the changes in cell and FA strains with the different FA stiffness and location differences; representative images were taken from the compliant substrate condition. (D) Median cell basal strain is highest in the $10 \mathrm{kPa}$ and distributed FA conditions; this pattern held true for all substrate conditions; strain values for part $\mathrm{D}$ are taken from 1,328 element integration points, error bars are 95\% confidence interval, \#\#\# $\mathrm{p}$ $<0.0005$, Kruskal-Wallis with Dunn's post-hoc.

\section{Cell strain heterogeneity increases with distributed and stiffer FAs (cell-FA-substrate models)}

Cell basal strain heterogeneity is also affected by the locations and stiffness of FAs. Histogram plots for the compliant substrate with $1 \mathrm{kPa} F A s$ demonstrate increased cell strain heterogeneity with distributed compared to grouped FAs (Fig 6A) and Lorentzian curves were fit to the histograms to quantitatively assess the spread of values (Fig 6B). These data demonstrate that the 
locations of the FAs can impact not only median cell strains (as shown in Fig 5D), but also the heterogeneity of the cell strain profiles. FA stiffness can also play a role in cell basal strain heterogeneity, as demonstrated by the histogram plots between 1 and $10 \mathrm{kPa}$ grouped FAs for a cell on a compliant substrate (Fig 6C). Lorentzian distributions of these plots (Fig 6D) demonstrate higher cell basal strain heterogeneity with stiff adhesions. Lorentzian width values were calculated for all substrate and FA conditions (Fig 6E). Overall, these data demonstrate that cell basal strain heterogeneities in the cell-FA-substrate models are impacted by FA locations and stiffness. 

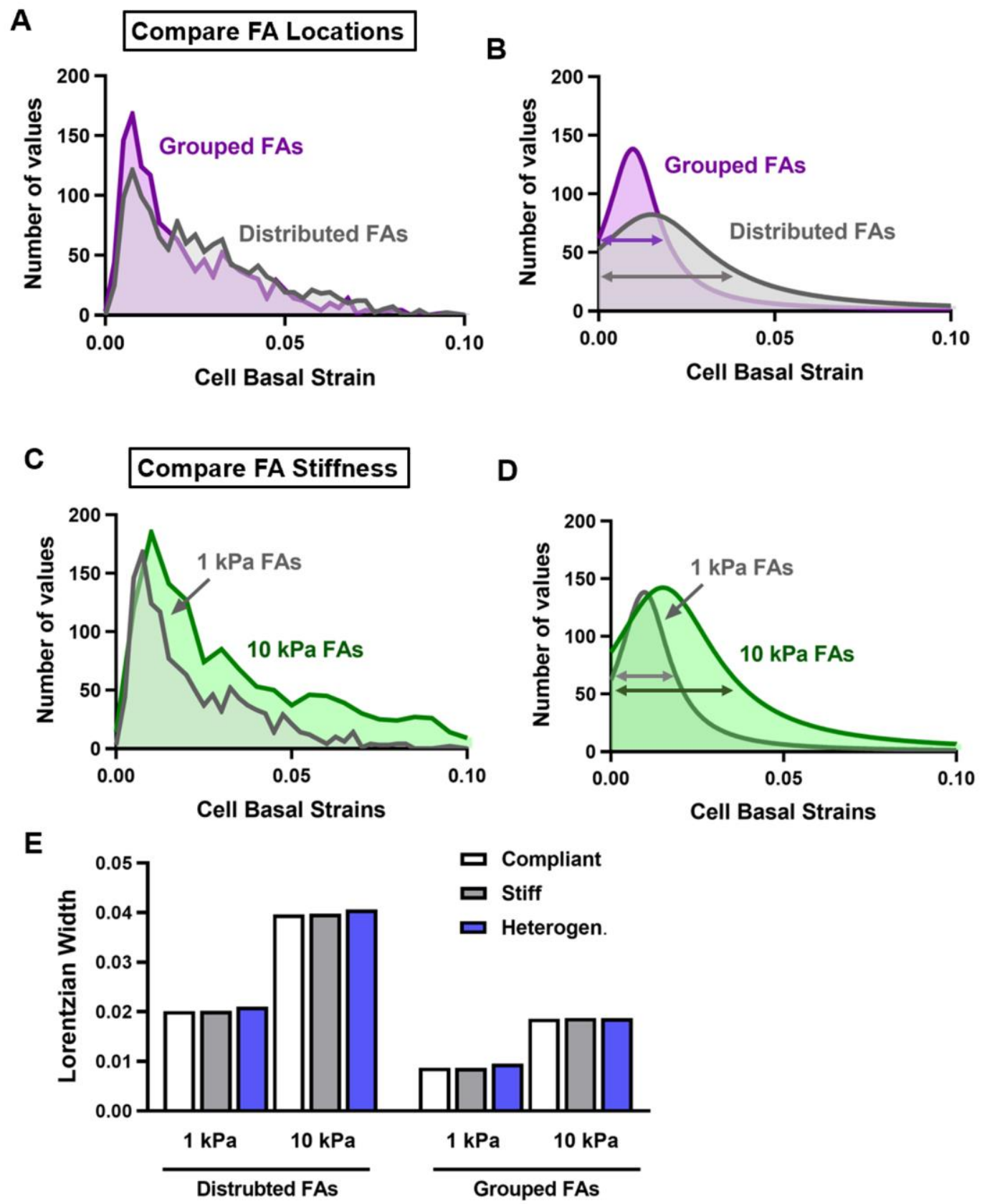

Fig 6: Impact of FA location and stiffness on cell basal strain heterogeneity. (A) Histogram profiles indicate a larger cell basal strain distribution with distributed compared to the grouped FAs. Representative data are from models including $1 \mathrm{kPa}$ FAs placed on the compliant substrate following shear stress and substrate stretch loading. (B) Lorentzian distributions were fit to the histogram profiles and used to quantitively measure the Lorentzian widths (indicated by arrows). (C) Histograms of cell strains are impacted by FA stiffness. Representative data are from the grouped FA models on the compliant substrate. (D) Lorentzian distributions of the histograms 
demonstrate the increased heterogeneities present in cell basal strains with $10 \mathrm{kPa}$ FAs; arrows represent the Lorentzian width values. (E) Lorentzian width values for all substrate and FA conditions, demonstrating increased cell basal strain heterogeneity in the $10 \mathrm{kPa}$ and distributed FAs.

\section{Effects of focal adhesion presence: comparing the contact models}

Median cell strains were lower in the model which included FAs compared to the model without FAs present; median cell basal strain values for the cell-substrate model ranged from $0.1364-0.1371$ (Fig 4B), whereas median cell strain values for the cell-FA-substrate model ranged from $\sim 0.016-0.043$ (Fig 5D). There were no significant effects in cell strain values due to substrate conditions in either contact model. FA presence also had an effect on cell strain heterogeneity. Whereas without FA contacts there was a $36.8 \%$ difference in Lorentzian width values from cell basal strain profiles between homogeneous compliant and heterogeneous substrates (Fig 4E), with the presence of FA contacts in the model, there are only modest changes between these two substrates: $4.2 \%$ for $1 \mathrm{kPa}$ distributed FAs; $2.5 \%$ for $10 \mathrm{kPa}$ distributed FAs; $8.7 \%$ for $1 \mathrm{kPa}$ grouped FAs; and $0.59 \%$ for $10 \mathrm{kPa}$ grouped FAs (Fig 7). Overall, these data demonstrate that cell basal strain heterogeneities are affected by substrate stiffness heterogeneities to a greater extent in the cell-substrate contact model than in the cellFA-substrate contact model. Therefore, the inclusion of FAs into the model has the effect of decreasing and homogenizing the cell strains.

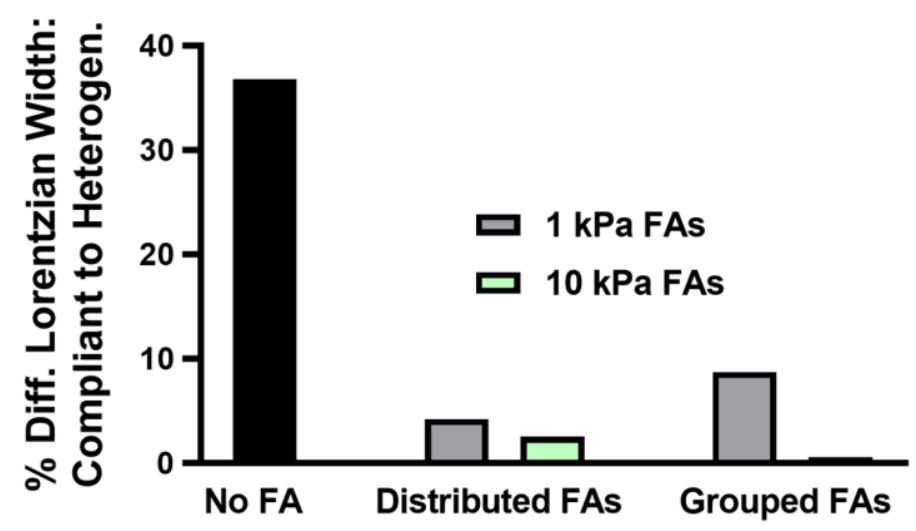

Fig 7: Cell basal strain heterogeneity is muted with the presence of FAs. Percent difference in cell basal strain heterogeneity (measured using Lorentzian curve widths) from cells placed on the 
compliant, homogenous substrate to the heterogeneous substrate for different FA conditions in response shear and substrate stretch loading.

\section{Discussion}

Our results demonstrate that the presence and properties of FAs in computational models can alter cell basal strains. FAs served to 'absorb' the substrate strain, causing mean cell strain to decrease substantially with the presence of FAs. In particular, more compliant FAs 'absorbed' more of the substrate strain, as cell strains were the lowest in the $1 \mathrm{kPa}$ FA conditions. We believe this is due to the fact that a compliant material deforms more when loaded with a given strain. Therefore, more strain was 'absorbed' by the compliant FAs than the stiff FAs, causing more strain to be 'passed onto' the cell in the stiff FA conditions. Addition of FAs also homogenized the cell basal strains; without FAs present in the model, there was a $36.8 \%$ difference in Lorentzian widths from homogeneous to heterogeneous substrates, but Lorentzian width values only changed $0.59-8.7 \%$ when FAs were included in the model. This may be due to the higher strains exhibited in the cell with the cell-substrate contact model compared to the cell-FA-substrate, with an order of magnitude difference. When FAs are not included in the model, the cell exhibits higher strains, and the changes in cell strain heterogeneities become more difficult to detect. Therefore, the overall presence of FAs serves to 'absorb' strains that would be transmitted to the cell if the FAs were absent.

Our model also considers micro-scale substrate stiffness changes along with different FA locations. It has been shown that cells exert higher traction forces and exhibit more monolayer disruption on stiff substrates [24,25], and that cells placed on a soft-stiff interface developed different local traction forces based on local rigidities [8]. In vitro, we previously demonstrated that cells place more FA contacts on stiff substrate regions, thereby causing FA groupings [3]. Here, we simulated this effect by using a checkerboard heterogeneous matrix, and chose to limit our analysis to heterogeneity changes on the $10 \mu \mathrm{m}$ length-scale, as was used in our previous $e x$ vivo study [7]. We found that grouped FAs cause more homogeneous cell strain profiles than distributed FAs. A previous cell-FA-substrate in silico model also suggested that cells may respond to mechanical cues by reaching a more homeostatic stress state [12]. Our data add to the 
previous literature by proposing the idea that cells configure their FAs to minimize mean cellular strain and achieve a more homogenous strain pattern.

Our work uses substrate stretch and shear stress as the loading conditions to understand EC response to substrate stiffness heterogeneities. In our model, substrate stretch had a greater impact on cell strain than apical shear stress. Although the effects from shear stress alone were minimal, we found it important to include this loading condition in our model to better simulate physiological conditions. Interestingly, the combined effects of substrate stiffness and stretch on cellular mechanotransduction has recently been studied in vitro. In a study by Quinlan et al. (2011), cells remained rounded on a compliant gel until induction of cyclic stretch, which caused them to spread to the size of cells on an undeformed stiff substrate. The authors propose that the mechanism of cellular mechanosensing may be similar for detecting substrate stiffness and substrate stretch [26]. This hypothesis was tested in a computational model by Parameswaran et al. (2014), which indicates that active reorganization of actomyosin structures allows for both substrate stiffness and stretch mechanotransduction by cells [27]. These studies, along with our results presented here, indicate the importance of understanding the effects of multiple mechanical cues on cellular mechanotransduction.

In our study of the cell-substrate model, shear stress was minimally impactful on cell strains, as values due to shear stress alone were an order of magnitude lower than those caused by substrate deformation. We expected a lower effect from fluid shear stress than from substrate stretch as cells form focal adhesion contacts with the underlying extracellular proteins, but not the blood on the apical surface. However, shear plays a vital role in vascular health through the mechanosensitivity of ECs and their proteins. Shear flow on an EC monolayer orients the direction of EC elongation [22], and can cause small cytoplasmic deformations [11]. We have previously demonstrated that the combined effects of shear stress and substrate rigidity affect EC junctional stability and nitric oxide production through changes in RhoA and eNOS activation [22]. Flow can also induce cytoskeletal remodeling and mediate the mechanical tension across cell-cell junctions through proteins such as platelet-endothelial cell adhesion molecule-1 (PECAM-1) and VE-cadherin [28]. Therefore, given the importance of shear stress in 
mechanical loading of ECs, our experiments were carried out under conditions of combined substrate stretch and shear stress.

Our model tries to mimic the in vivo environment to help analyze changes in EC strain profiles due to FA locations on a heterogeneous substrate. However, not all conditions can be included, and therefore the model does have limitations. In our simulated substrate, we assumed heterogeneity in distinct squares, and a flat surface. This simulated substrate does not accurately mimic the fibrous and extremely heterogeneous nature of the extracellular matrix (ECM) in vivo. Previous work has demonstrated that the fibrous nature of the ECM has implications on cell signaling [29]. In addition, here we used a cell with a distinct nucleus stiffness, but other organelles and cytoskeletal fibers were not included in the model. For the loading conditions, we also assumed a uniform, non-pulsatile apical shear on the EC and a uniform substrate strain. Also, while the cell was placed individually, ECs in vivo are found in a cell monolayer in the artery intima. This model therefore has multiple avenues to pursue in the future to make it as physiologically representative as possible. Finally, previous in vitro studies have examined strain changes due to exogeneous forces in EC cytoskeletal networks [30] and in FA proteins themselves [31]. While we believe that developing an in vitro model of these conditions would be beneficial, this is beyond the scope of this manuscript.

\section{Conclusions}

A finite element model was created to assess the impact of micro-scale stiffness heterogeneities and focal adhesions in concert on endothelial cell strains. The presence of focal adhesions in the model reduced the mean cell strains and served to homogenize the cell strain profiles, demonstrating the importance of including focal adhesions into in silico models of cell-substrate interactions. In addition, cell strains were decreased and more homogeneous with more compliant FAs and when FAs were grouped onto stiffer substrate regions. We propose that cells may respond to mechanical stimuli with particular FA configurations in an effort to decrease and homogenize cellular strains. 


\section{Funding}

This research was supported by the National Research Foundation (NRF) of South Africa (UID 93542), and the South African Medical Research Council (MRC) under a Self-initiated Research

Grant (SIR 328148). Views and opinions expressed are not those of the NRF or SAMRC but of the authors. Further support was provided by the National Science Foundation in the United States of America (grant 1738345). Support for J.C.K. was provided by the Whitaker International Program.

\section{Conflict of Interest}

The authors declare that they do not have conflicts of interest.

\section{References}

1. Burridge K, Fath K, Kelly T, Nuckolls G, Turner C. Focal adhesions: transmembrane junctions between the extracellular matrix and the cytoskeleton. Annu Rev Cell Biol. 1988;4: 487-525. doi:10.1146/annurev.cb.04.110188.002415

2. Paszek MJ, Zahir N, Johnson KR, Lakins JN, Rozenberg GI, Gefen A, et al. Tensional homeostasis and the malignant phenotype. Cancer Cell. 2005;8: 241-254. doi:10.1016/j.ccr.2005.08.010

3. Lampi MC, Guvendiren M, Burdick JA, Reinhart-King CA. Photopatterned Hydrogels to Investigate Endothelial Cell Response to Matrix Stiffness Heterogeneity. ACS Biomater Sci Eng. 2017;3: 3007-3016. doi:10.1021/acsbiomaterials.6b00633

4. Owen G, Meredith D, ap Gwynn I, Richards R. Focal adhesion quantification- a new assay of material biocompatibility?: Review. Eur Cells Mater. 2005;9: 85-96. doi:10.22203/eCM.v009a10

5. Han SJ, Bielawski KS, Ting LH, Rodriguez ML, Sniadecki NJ. Decoupling substrate stiffness, spread area, and micropost density: a close spatial relationship between traction forces and focal adhesions. Biophys J. 2012;103: 640-648. doi:10.1016/j.bpj.2012.07.023

6. Byfield FJ, Reen RK, Shentu TP, Levitan I, Gooch KJ. Endothelial actin and cell stiffness is modulated by substrate stiffness in 2D and 3D. J Biomech. 2009;42: 1114-1119. doi:10.1016/j.jbiomech.2009.02.012

7. Kohn JC, Chen A, Cheng S, Kowal DR, King MR, Reinhart-King CA. Mechanical 
heterogeneities in the subendothelial matrix develop with age and decrease with exercise. J Biomech. Elsevier; 2016;49: 1447-1453. doi:10.1016/j.jbiomech.2016.03.016

8. Breckenridge MT, Desai RA, Yang MT, Fu J, Chen CS. Substrates with engineered step changes in rigidity induce traction force polarity and durotaxis. Cell Mol Bioeng. 2014;7: 26-34. doi:10.1007/s12195-013-0307-6

9. Krishnan R, Oommen B, Walton EB, Maloney JM, Van Vliet KJ. Modeling and simulation of chemomechanics at the cell-matrix interface. Cell Adh Migr. 2008;2: 83-94. doi:10.4161/cam.2.2.6154

10. Hu S, Chen J, Fabry B, Numaguchi Y, Gouldstone A, Ingber DE, et al. Intracellular stress tomography reveals stress focusing and structural anisotropy in cytoskeleton of living cells. Am J Physiol Cell Physiol. 2003;285: C1082-C1090.

doi:10.1152/ajpcell.00159.2003

11. Ferko MC, Bhatnagar A, Garcia MB, Butler PJ. Finite-element stress analysis of a multicomponent model of sheared and focally-adhered endothelial cells. Ann Biomed Eng. 2007;35: 208-223. doi:10.1007/s10439-006-9223-4

12. Mullen CA, Vaughan TJ, Voisin MC, Brennan MA, Layrolle P, McNamara LM. Cell morphology and focal adhesion location alters internal cell stress. J R Soc Interface. 2014;11: 20140885. doi:10.1098/rsif.2014.0885

13. Abdalrahman T, Dubuis L, Green J, Davies N, Franz T. Cellular mechanosensitivity to substrate stiffness decreases with increasing dissimilarity to cell stiffness. Biomech Model Mechanobiol. Springer Berlin Heidelberg; 2017;16: 2063-2075. doi:10.1007/s10237-0170938-y

14. Kohn JC, Abdalrahman T, Sack KL, Reinhart-King CA, Franz T. Endothelial cells on an aged subendothelial matrix display heterogeneous strain profiles in silico. Biomech Model Mechanobiol. Springer Berlin Heidelberg; 2018;17: 1405-1414. doi:10.1007/s10237-0181034-7

15. Cao X, Lin Y, Driscoll T, Franco-Barraza J, Cukierman E, Mauck R, et al. A chemomechanical model of matrix and nuclear rigidity regulation of focal adhesion size. Biophys J. Biophysical Society; 2015;109: 1807-1817. doi:10.1016/j.bpj.2015.08.048

16. Oakes PW, Gardel ML. Stressing the limits of focal adhesion mechanosensitivity. Curr Opin Cell Biol. Elsevier Ltd; 2014;30: 68-73. doi:10.1016/j.ceb.2014.06.003 
17. Engler A, Bacakova L, Newman C, Hategan A, Griffin M, Discher D. Substrate compliance versus ligand density in cell on gel responses. Biophys J. 2004;86: 617-628. doi:10.1016/S0006-3495(04)74140-5

18. Nicolas A, Safran S a. Limitation of cell adhesion by the elasticity of the extracellular matrix. Biophys J. Elsevier; 2006;91: 61-73. doi:10.1529/biophysj.105.077115

19. Caille N, Thoumine O, Tardy Y, Meister J-J. Contribution of the nucleus to the mechanical properties of endothelial cells. J Biomech. 2002;35: 177-187. doi:10.1016/S0021-9290(01)00201-9

20. Yao Y, Lacroix D, Mak AFT. Effects of oxidative stress-induced changes in the actin cytoskeletal structure on myoblast damage under compressive stress: confocal-based cellspecific finite element analysis. Biomech Model Mechanobiol. Springer Berlin Heidelberg; 2016;15: 1-14. doi:10.1007/s10237-016-0779-0

21. Trachet B, Fraga-Silva RA, Londono FJ, Swillens A, Stergiopulos N, Segers P. Performance comparison of ultrasound-based methods to assess aortic diameter and stiffness in normal and aneurysmal mice. PLoS One. 2015;10: e0129007. doi:10.1371/journal.pone.0129007

22. Kohn JC, Zhou DW, Bordeleau F, Zhou AL, Mason BN, Mitchell MJ, et al. Cooperative effects of matrix stiffness and fluid shear stress on endothelial cell behavior. Biophys J. 2015;108: 471-478. doi:10.1016/j.bpj.2014.12.023

23. Weisstein EW. Lorentzian Function. In: MathWorld--A Wolfram Web Resource [Internet]. [cited 1 May 2019]. Available:

http://mathworld.wolfram.com/LorentzianFunction.html

24. Huynh J, Nishimura N, Rana K, Peloquin JM, Califano JP, Montague CR, et al. Agerelated intimal stiffening enhances endothelial permeability and leukocyte transmigration. Sci Transl Med. 2011;3: 112ra122. doi:10.1126/scitranslmed.3002761

25. Krishnan R, Klumpers DD, Park CY, Rajendran K, Trepat X, van Bezu J, et al. Substrate stiffening promotes endothelial monolayer disruption through enhanced physical forces. Am J Physiol Cell Physiol. 2011;300: C146-C154. doi:10.1152/ajpcell.00195.2010

26. Quinlan AM, Sierad LN, Capulli AK, Firstenberg LE, Billiar KL. Combining dynamic stretch and tunable stiffness to probe cell mechanobiology in vitro. PLoS One. 2011;6: e23272. doi:10.1371/journal.pone.0023272 
27. Parameswaran H, Lutchen KR, Suki B. A computational model of the response of adherent cells to stretch and changes in substrate stiffness. J Appl Physiol. 2014;116: 825834. doi:10.1152/japplphysiol.00962.2013

28. Conway DE, Breckenridge MT, Hinde E, Gratton E, Chen CS, Schwartz MA. Fluid shear stress on endothelial cells modulates mechanical tension across VE-cadherin and PECAM-1. Curr Biol. Elsevier Ltd; 2013;23: 1024-1030. doi:10.1016/j.cub.2013.04.049

29. Baker BM, Trappmann B, Wang WY, Sakar MS, Iris L, Shenoy VB, et al. Cell-mediated fiber recruitment drives extracellular matrix mechanosensing in engineered fibrillar microenvironments. Nat Mater. 2015;14: 1262-1268. doi:10.1038/nmat4444.Cellmediated

30. Helmke BP, Rosen AB, Davies PF. Mapping mechanical strain of an endogenous cytoskeletal network in living endothelial cells. Biophys J. Elsevier; 2003;84: 2691-2699. doi:10.1016/S0006-3495(03)75074-7

31. Sigaut L, Bilderling C Von, Bianchi M, Burdisso JE, Gastaldi L, Pietrasanta LI. Live cell imaging reveals focal adhesions mechanoresponses in mammary epithelial cells under sustained equibiaxial stress. Sci Rep. 2018;8: 9788. doi:10.1038/s41598-018-27948-3 